\title{
Exploring the Higgs portal
}

\author{
Christoph Englert, ${ }^{1}$ Tilman Plehn, ${ }^{1}$ Dirk Zerwas, ${ }^{2}$ and Peter M. Zerwas ${ }^{3,4}$ \\ ${ }^{I}$ Institut für Theoretische Physik, Universität Heidelberg, Germany \\ ${ }^{2} L A L, I N 2 P 3 / C N R S$, Orsay, France \\ ${ }^{3}$ Deutsches Elektronen-Synchrotron DESY, Hamburg, Germany \\ ${ }^{4}$ Institut für Theoretische Teilchenphysik und Kosmologie, RWTH Aachen University, Germany
}

(Dated: June 15, 2011)

\begin{abstract}
We study the Higgs portal from the Standard-Model to a hidden sector and examine which elements of the extended theory can be discovered and explored at the LHC. Our model includes two Higgs bosons covering parameter regions where the $\mathrm{LHC}$ will be sensitive to two, one or none of the particles at typical discovery luminosities for Standard Model Higgs production.
\end{abstract}

\section{INTRODUCTION}

Theoretical scenarios beyond the Standard Model [SM] which will be tested at the LHC [1] often include a hidden sector. The standard sector and the hidden sector are coupled by interactions of gauge-invariant operators which open the gate for exploring structures in the hidden sector by observing phenomena in the visible standard sector. An attractive realization of this idea is provided by the Higgs portal which connects the Higgs fields in the two sectors by an elementary quartic interaction [2-14]. Such a setup moves a precision study of the Higgs sector [15-17] into a central position of new physics searches at the LHC.

Starting from a wide range of Higgs observables at the LHC $[18,19]$ its focus will naturally be on measurements of Higgs masses, couplings and, to a lesser extent, Higgs self-interactions particularly in cascade decays [20]. The key observables which allow for such an analysis are production rates for different decay channels combined with the weak boson fusion process [21, 22] and the recently revived associated production channels with decays to bottom pairs [23]. For Higgs masses between 120 and $160 \mathrm{GeV}$ the LHC, running at a center-of-mass energy of $14 \mathrm{TeV}$ and collecting integrated luminosities in the $\mathcal{O}\left(10-100 \mathrm{fb}^{-1}\right)$ range, can detect uncorrelated modifications to individual Higgs couplings of the order of $30 \%$ to 50\% [16]. Provided there exists some kind of universal pattern in these modified couplings, the sensitivity increases to $20 \%$ or better [13]. A Higgs portal or hidden Higgs sector is such a case with generally well-defined patterns in the modified couplings. To render our analysis as transparent as possible, we will illustrate the basic idea in a set-up in which fields and interactions are isomorphic in the two sectors, just supplemented by the quartic portal interaction. Adapting our results to other models should be straightforward.

We study the set of observables of a hidden Higgs sector and examine to which extent it can be reconstructed by precision measurements in collider experiments, $n . b$. at the LHC. In an earlier, related study [13] we restricted ourselves to the properties of the SM-type Higgs boson. To explore elements of the hidden sector, invisible Higgs decays [24] to particles in this sector play a crucial role. Valuable additional insight we can obtain from standard Higgs properties like masses and visible decay branching ratios. The fundamental question whether a Higgs portal with noticeable interactions between standard and hidden sector exists or not, can be answered this way.

In this extended analysis we systematically explore the maximum information that can be obtained on the Higgs portal and the associated standard and hidden sector states from established Higgs search strategies [25], i.e. we consider both Higgs masses lighter than $\mathcal{O}(1 \mathrm{TeV})$. For Higgs spectra with the heavy narrow mass state in the trans-TeV region, analysis strategies have been described in Ref. [3]; discovery reaches for broad and heavy states are discussed in, e.g., Ref. [26]. The key question is how we can link the parameters in our Higgs potential to general observables, like masses, cross sections, or decay widths, and then to possible LHC measurements, like twin width ratios [13]. While this work is not meant to be an experimental analysis, realistically modelling all statistical, systematic, and theoretical uncertainties, it defines the strategy underlying such an analysis and points out its critical steps from an experimental and theoretical point of view.

\section{FROM THE POTENTIAL TO COLLIDER OBSERVABLES}

Before we discuss realistic LHC prospects, it is important to study the structure of Higgs portal models and identify the complete set of observables which we can then try and access at the LHC. The Higgs potential we study in this letter consists of the Standard Model component $[s]$, the isomorphic component in the hidden sector $[h]$, and the quartic interaction coupling the two sectors with strength $\eta_{\chi}$, videlicet,

$$
\mathcal{V}=\mu_{s}^{2}\left|\phi_{s}\right|^{2}+\lambda_{s}\left|\phi_{s}\right|^{4}+\mu_{h}^{2}\left|\phi_{h}\right|^{2}+\lambda_{h}\left|\phi_{h}\right|^{4}+\eta_{\chi}\left|\phi_{s}\right|^{2}\left|\phi_{h}\right|^{2} .
$$

The mass parameters $\mu_{j}$ are generally substituted by $v_{j}$ after expanding the two Higgs fields about their vacuum expectation values, $\phi_{j} \rightarrow\left(v_{j}+H_{j}\right) / \sqrt{2}$ with $v_{j}^{2}=\left(-\mu_{j}^{2}-\eta_{\chi} v_{i}^{2} / 2\right) / \lambda_{j}$ for $i \neq j=s, h$. The SM Higgs vacuum expectation value is 
fixed by the gauge boson masses, since even in the presence of a non-vanishing expectation value the hidden Higgs fields do not contribute to electroweak symmetry breaking in the standard sector. This is an important difference between the hidden Higgs sector and other multi-Higgs models. However, due to the coupling of the two sectors the physical Higgs states in the SM and the hidden sector mix to the mass eigenstates

$$
\begin{aligned}
& H_{1}=\cos \chi H_{s}+\sin \chi H_{h} \\
& H_{2}=-\sin \chi H_{s}+\cos \chi H_{h} .
\end{aligned}
$$

Both, $H_{1}$ and $H_{2}$, couple to Standard Model fields through their components $H_{s}$ and to the hidden sector through the admixtures $H_{h}$. For moderate coupling $\eta_{\chi}$ the properties of $H_{1}$ remain dominated by the Standard Model component, while the properties of $H_{2}$ are characterized primarily by the hidden Higgs component. The mixing of the fields in the potential generates selfinteractions among the light and heavy Higgs bosons [3], in particular trilinear couplings $H_{i} H_{j} H_{k}$ of any combination.

The phenomenology of the Higgs portal to the hidden sector depends on the ratio of the Higgs boson masses. We will take $H_{1}$, primarily $[s]$, to be the lighter particle and $H_{2}$, primarily [h], to be the heavier companion. Any scenario with other mass ratios could be treated analogously but suffers from electroweak precision and unitarity constraints.

The properties of the two Higgs bosons are summarized in the masses, $M_{1,2}$, the visible and invisible widths, $\Gamma_{1,2}^{\mathrm{vis}}$ and $\Gamma_{1,2}^{\mathrm{inv}}$, both defined without including Higgs cascade decays, and finally the Higgs cascade $\Gamma_{2}^{H} H$, realized by $H_{2} \rightarrow H_{1} H_{1}$ for suitable mass ratios. From these observables we can derive all fundamental parameters of the Higgs potential.

(i) Higgs masses — Diagonalizing the Higgs mass matrix [squared],

$$
\mathcal{M}^{2}=\left(\begin{array}{cc}
2 \lambda_{s} v_{s}^{2} & \eta_{\chi} v_{s} v_{h} \\
\eta_{\chi} v_{s} v_{h} & 2 \lambda_{h} v_{h}^{2}
\end{array}\right)
$$

generates the mass eigenvalues $M_{1,2}$ and the mixing angle $\chi$,

$$
\begin{aligned}
M_{1,2}^{2} & =\left[\lambda_{s} v_{s}^{2}+\lambda_{h} v_{h}^{2}\right] \mp\left|\lambda_{s} v_{s}^{2}-\lambda_{h} v_{h}^{2}\right| \sqrt{1+\tan ^{2} 2 \chi} \\
\tan 2 \chi & =\frac{\eta_{\chi} v_{s} v_{h}}{\lambda_{s} v_{s}^{2}-\lambda_{h} v_{h}^{2}} \quad \text { with } \quad \pi / 8 \leq \pm \chi \pm \pi / 8 \leq 3 \pi / 8,
\end{aligned}
$$

for the two mass eigenstates $H_{1,2}$ defined in Eq. (2). The sign in front of $\chi$ coincides with $\operatorname{sgn}\left[\eta_{\chi}\right]$ while the sign of the phase shift $\pm \pi / 8$ corresponds to $\operatorname{sgn}\left[\lambda_{s} v_{s}^{2}-\lambda_{h} v_{h}^{2}\right]$. The mixing is restricted to

$$
\tan ^{2} 2 \chi \leq \frac{4 \lambda_{s} \lambda_{h} v_{s}^{2} v_{h}^{2}}{\left[\lambda_{s} v_{s}^{2}-\lambda_{h} v_{h}^{2}\right]^{2}} .
$$

For $v_{h}=v_{s}=246 \mathrm{GeV}$ and $\lambda_{s}=\lambda_{h} / 4=1 / 8$, a parameter set reminiscent of the Standard Model, we illustrate the two Higgs masses as functions of the mixing parameter in Fig. 1. They are compared with the bounds derived from the non-observation of Higgs bosons at LEP for standard and reduced couplings [27]. For this illustrational parameter set the mixing has to stay below $\sin \chi \leq 0.22$, as a direct result of the LEP bound on the Higgs mass. This kind of bound is a general feature, because the mixture of a Standard Model and a relatively light hidden Higgs state will generate one mass eigenvalue below the SM diagonal entry in Eq. (4).

Unitarity for high energies and precision observables like the $\rho$ parameter constrain the mass values in complete analogy to the Standard Model case. The usual SM Higgs mass or its logarithm is substituted by the superposition of the two Higgs masses, weighted by the mixing parameters $\cos ^{2} \chi$ and $\sin ^{2} \chi$, e.g.,

$$
\begin{array}{lrl}
\text { unitarity } & M_{H_{\mathrm{SM}}}^{2} & \rightarrow\left\langle M_{i}^{2}\right\rangle \equiv \cos ^{2} \chi M_{1}^{2}+\sin ^{2} \chi M_{2}^{2} \leq 4 \pi \sqrt{2} / 3 G_{F} \simeq(700 \mathrm{GeV})^{2} \\
\rho \text { parameter } & \log M_{H_{\mathrm{SM}}}^{2} & \rightarrow\left\langle\log M_{i}^{2}\right\rangle \equiv \cos ^{2} \chi \log M_{1}^{2}+\sin ^{2} \chi \log M_{2}^{2} \leq \log (175 \mathrm{GeV})^{2} .
\end{array}
$$

As expected, for small mixing the SM bounds transfer to $M_{1}$ while $M_{2}$ remains essentially unconstrained. However, for large mixing the two bounds transfer to the algebraic and geometric means of the $M_{1}, M_{2}$ mass pair, thus reducing the allowed range for $M_{2}$ considerably. Finally, for large $\sin ^{2} \chi, M_{1}$ and $M_{2}$ interchange their roles.

Because the most restrictive bounds arise from electroweak precision data we base our numerical scan over the Higgs potential on the complete set of $S, T, U$ parameters [28]. Confronting the model defined in Eq. (1) with the current bounds on $S, T, U$ [29], 

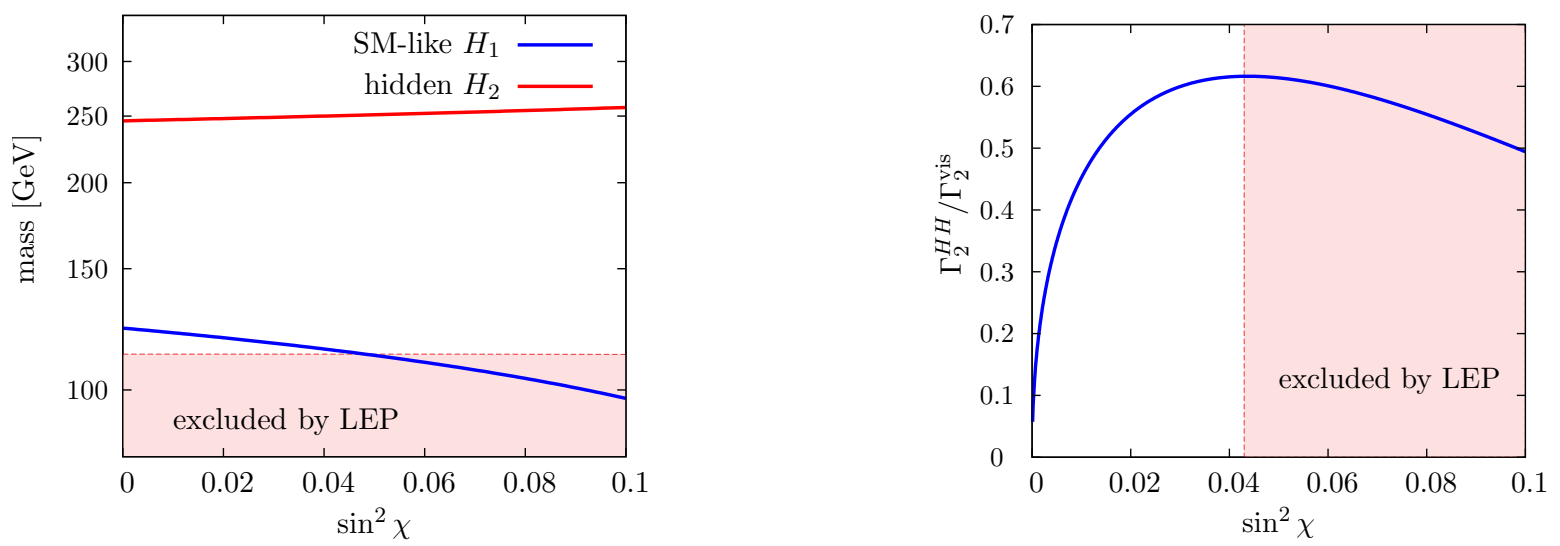

FIG. 1: Left: masses of the light SM-like Higgs boson $H_{1}$ (blue) and the heavy Higgs boson $H_{2}$ (red). The parameters of the Higgs potentials are $v_{h}=v_{s}=246 \mathrm{GeV}$ and $\lambda_{s}=\lambda_{h} / 4=1 / 8$. The shaded region displays the LEP bound [27]. Right: cascade decay width $\Gamma_{2}^{H H}$ as a function of $\sin ^{2} \chi$ for the same parameters. Again, the region in which $H_{1}$ is excluded by LEP is shaded.

we need to emphasize one caveat: if the spontaneous symmetry breaking in the hidden sector also gives rise to additional massive states, the $S, T, U$ values [3, 30,31] may be altered significantly, e.g., through kinetic $U(1)$ mixing [32]. Since we are primarily interested in the phenomenology of the two Higgs states, Eq. (4), we do not further investigate this direction, yet we also consider parameter points of the portal model which violate the bounds by about $10 \%$.

(ii) Direct Higgs production and decay - All $H_{1}$ couplings to SM-particles are suppressed by the mixing parameter $\cos \chi, H_{2}$ couplings correspondingly by $\sin \chi$; for direct decays to particles in the hidden sector the two coefficients are reversed:

$$
\begin{array}{lll}
\Gamma_{1}^{\mathrm{vis}}=\cos ^{2} \chi \Gamma_{1}^{\mathrm{SM}} & \text { and } & \Gamma_{2}^{\mathrm{vis}}=\sin ^{2} \chi \Gamma_{2}^{\mathrm{SM}} \\
\Gamma_{1}^{\mathrm{inv}}=\sin ^{2} \chi \Gamma_{1}^{\mathrm{hid}} & \text { and } & \Gamma_{2}^{\text {inv }}=\cos ^{2} \chi \Gamma_{2}^{\text {hid }} .
\end{array}
$$

While $\Gamma_{1,2}^{\mathrm{SM}}$ denote the SM Higgs widths for the eigenstates with masses $M_{1,2}, \Gamma_{1,2}^{\text {hid }}$ play the same role in the hidden sector, i.e. Higgs cascade decays $H_{2} \rightarrow H_{1} H_{1}$ not included. The same index 'vis' may also specify a subset of final states. The cross sections for Higgs production channels in all SM-particle collisions are affected analogously

$$
\sigma_{1}=\cos ^{2} \chi \sigma_{1}^{\mathrm{SM}} \quad \text { and } \quad \sigma_{2}=\sin ^{2} \chi \sigma_{2}^{\mathrm{SM}} .
$$

At hadron colliders, we measure a particular combination of partial widths and production cross sections as the twin-width ratios [13]

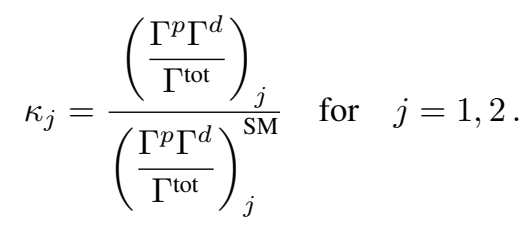

The partial widths refer to the production channel $p$ and the decay mode $d$, either exclusively or summed over sets of initial or final states. These ratios are measured, at the Born level, directly by the product of production cross section times decay branching ratio of the process $p \rightarrow H i g g s \rightarrow d$ in the narrow-width approximation. The parameters $\kappa_{1,2}$ are independent of $p, d$ with values less than unity, and any pair $[p, d]$ may be exploited to measure $\kappa_{j}$. The universality of the parameters $\kappa_{1,2}$ is a signal for mixed Higgs states or a universal coupling modification due to a naive bound state form factor [13, 33]. Joining the measurement of the $\kappa_{j}$ with the invisible branching ratios allows us to determine the mixing parameter $\sin ^{2} \chi$.

(iii) Invisible Higgs decay - For $H_{1}$ the invisible decay width is generated merely by direct decays into the hidden sector, for $\overline{H_{2}}$ the direct decays may be supplemented by cascades. For this argument we assume that the standard Higgs decays $H \rightarrow Z Z \rightarrow 4 \nu$ are subtracted after measuring the corresponding $4 \ell$ modes. For light states $H_{1}$ there is a non-negligible decay rate of the Higgs into gluons and charm quarks. These branching ratios are phenomenologically inaccessible at the LHC due to large backgrounds. This adds to complications in extracting the Higgs-to-invisible branching ratio, which are already familiar from the SM scenario. Two methods, consistent with the present portal scenario, can be followed to solve the problem. Either these modes may be included theoretically into the corpus of visible final states by adopting the SM couplings, as assumed in 
the model. Or the issue can be treated consistently by fitting a partial width for the undetected decays as part of a global fit to the model as described in Refs. $[15,16]$. In relation to the visible decay widths the invisible decay widths can be extracted by measuring the ratios of the corresponding branching ratios, i.e.

$$
\begin{aligned}
\frac{\Gamma_{1}^{\mathrm{inv}}}{\Gamma_{1}^{\mathrm{vis}}} & =\frac{\mathrm{BR}_{1}^{\mathrm{inv}}}{\mathrm{BR}_{1}^{\mathrm{vis}}}, \\
\frac{\Gamma_{2}^{\mathrm{inv}}+\Gamma_{2}^{H H, \text { inv }}}{\Gamma_{2}^{\mathrm{vis}}} & =\frac{\mathrm{BR}_{2}^{\text {inv }}}{\mathrm{BR}_{2}^{\mathrm{vis}}},
\end{aligned}
$$

where $\Gamma^{H H \text {,inv }}$ denotes the invisible decay width resulting from the cascade decay $H_{2} \rightarrow H_{1} H_{1}$ with invisible $H_{1}$ decay modes. In contrast to the ad-hoc definition of the visible branching ratio $\mathrm{BR}_{2}^{\text {vis }}=\Gamma_{2}^{\text {vis }} / \Gamma_{2}^{\text {tot }}$, the measured invisible branching ratio $\mathrm{BR}_{2}^{\text {inv }}$ necessarily includes the invisible cascade decays.

(iv) Higgs cascade - If $H_{2}$ is sufficiently heavier than $H_{1}$, the cascade channel $H_{2} \rightarrow H_{1} H_{1}$ opens up with its partial width

$$
\Gamma_{2}^{H H}=\frac{\Lambda_{211}^{2}}{32 \pi} \frac{\beta_{1}}{M_{2}}
$$

The velocity of $H_{1}$ in the rest frame of $H_{2}$ is denoted by $\beta_{1}$ while the effective $H_{2} H_{1} H_{1}$ coupling, derived by inserting the mixed states into the potential $\mathcal{V}$, reads

$$
\begin{aligned}
\Lambda_{211}= & 3 \sin 2 \chi\left[\cos \chi \frac{\lambda_{s} v_{s}^{2}}{v_{s}}-\sin \chi \frac{\lambda_{h} v_{h}^{2}}{v_{h}}\right] \\
& -\tan 2 \chi\left[\lambda_{s} v_{s}^{2}-\lambda_{h} v_{h}^{2}\right]\left[\left(1-3 \cos ^{2} \chi\right) \frac{\sin \chi}{v_{h}}-\left(1-3 \sin ^{2} \chi\right) \frac{\cos \chi}{v_{s}}\right] .
\end{aligned}
$$

The decays of the $H_{1} H_{1}$ pair give rise to visible-visible, visible-invisible, and invisible-invisible final states with probabilities $\cos ^{4} \chi, 2 \sin ^{2} \chi \cos ^{2} \chi$ and $\sin ^{4} \chi$, respectively. As a result, we can reconstruct $\Gamma_{2}^{H}{ }^{H}$ from the channel in which both $H_{1}$ decays are visible: $\Gamma_{2}^{H H}=\Gamma_{2}^{H H, v i s} / \cos ^{4} \chi$. To illustrate the probability of cascade decays we show the $\chi$-dependence of the ratio $\Gamma_{2}^{H H} / \Gamma_{2}^{\text {vis }}$ in the right panel of Fig. 1 .

From all observables listed above we can derive the fundamental properties of the two Higgs bosons, which are related to the dynamics in the hidden sector: the mixing angle $\sin \chi$, the invisible partial widths $\Gamma_{1,2}^{\text {inv }}$, the cascade width $\Gamma_{2}^{H H}$ and the total widths $\Gamma_{1,2}^{\text {tot }}$. The latter are notorious at hadron colliders. While we cannot experimentally determine them for narrow states, they are crucial properties of our two Higgs states. Provided $H_{2}$ is heavier than twice the $H_{1}$ mass, the total widths of the two Higgs bosons are given in terms of five partial widths

$$
\begin{aligned}
& \Gamma_{1}^{\mathrm{tot}}=\cos ^{2} \chi \Gamma_{1}^{\mathrm{SM}}+\sin ^{2} \chi \Gamma_{1}^{\mathrm{hid}} \\
& \Gamma_{2}^{\mathrm{tot}}=\sin ^{2} \chi \Gamma_{2}^{\mathrm{SM}}+\cos ^{2} \chi \Gamma_{2}^{\mathrm{hid}}+\Gamma_{2}^{H H} .
\end{aligned}
$$

Ratios of partial and total Higgs widths, however, are observable at the LHC. For the light SM-type Higgs boson the relations

$$
\begin{array}{ll}
\frac{\Gamma_{1}^{\mathrm{inv}}}{\Gamma_{1}^{\mathrm{SM}}}=\cos ^{2} \chi\left[\frac{\cos ^{2} \chi}{\kappa_{1}}-1\right], & \frac{\Gamma_{1}^{H H}}{\Gamma_{1}^{\mathrm{SM}}}=0, \\
\frac{\Gamma_{1}^{\mathrm{inv}}}{\Gamma_{1}^{\mathrm{SM}}}=\cos ^{2} \chi \frac{\mathrm{BR}_{1}^{\mathrm{inv}}}{\mathrm{BR}_{1}^{\mathrm{vis}}}, & \frac{\Gamma_{1}^{\mathrm{tot}}}{\Gamma_{1}^{\mathrm{SM}}}=\frac{\cos ^{4} \chi}{\kappa_{1}} .
\end{array}
$$

link the decay width to SM particles $\Gamma_{1}^{S M}$ to the total width $\Gamma^{\text {tot }}$ and provide us with two equivalent expressions for the modified invisible branching ratio. Thus, we can express the mixing angle $\cos ^{2} \chi$ and $\Gamma_{1}^{\text {inv }} / \Gamma_{1}^{S M}$ in terms of the observable twin-width ratio $\kappa_{1}$ and the branching ratios $\mathrm{BR}_{1}^{\text {inv }} / \mathrm{BR}_{1}^{\text {vis }}$.

The corresponding expressions for the heavy hidden-type Higgs boson $H_{2}$ are slightly modified because they include the cascade decay, followed by the decay of the $H_{1}$ pair back to the visible sector

$$
\begin{array}{ll}
\frac{\Gamma_{2}^{\mathrm{inv}}}{\Gamma_{2}^{\mathrm{SM}}}=\sin ^{2} \chi\left[\frac{\sin ^{2} \chi}{\kappa_{2}}-1-\frac{1}{\cos ^{4} \chi} \frac{\mathrm{BR}_{2}^{H H, \mathrm{vis}}}{\mathrm{BR}_{2}^{\mathrm{vis}}}\right], & \frac{\Gamma_{2}^{H H}}{\Gamma_{2}^{\mathrm{SM}}}=\frac{\sin ^{2} \chi}{\cos ^{4} \chi} \frac{\mathrm{BR}_{2}^{H H, \text { vis }}}{\mathrm{BR}_{2}^{\text {vis }},} \\
\frac{\Gamma_{2}^{\mathrm{inv}}}{\Gamma_{2}^{\mathrm{SM}}}=\sin ^{2} \chi\left[\frac{\mathrm{BR}_{2}^{\mathrm{inv}}}{\mathrm{BR}_{2}^{\mathrm{vis}}}-\tan ^{4} \chi \frac{\mathrm{BR}_{2}^{H H, \mathrm{vis}}}{\mathrm{BR}_{2}^{\mathrm{vis}}}\right], & \frac{\Gamma_{2}^{\mathrm{tot}}}{\Gamma_{2}^{\mathrm{SM}}}=\frac{\sin ^{4} \chi}{\kappa_{2}} .
\end{array}
$$



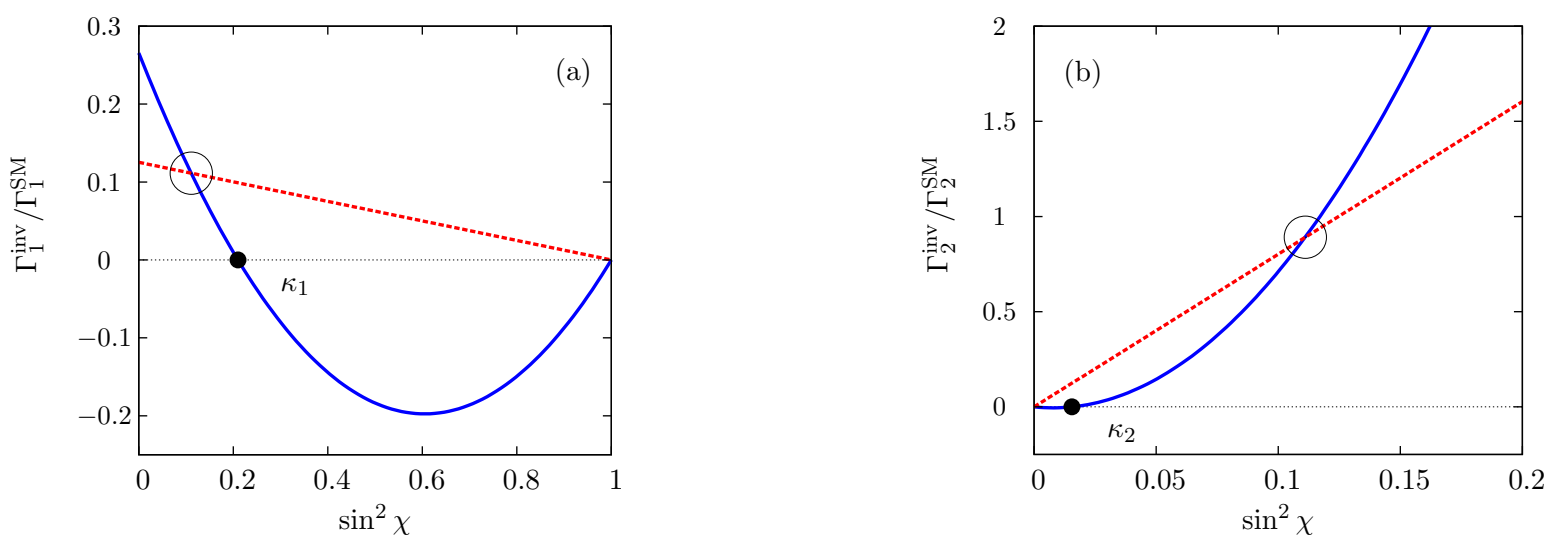

FIG. 2: Illustration of the intersections of the solutions for $\kappa_{j}$ (blue) and the invisible branching ratios (red), generating the physical solutions for the mixing parameter and the invisible widths. The Higgs portal model parameters are chosen $v_{s}=246 \mathrm{GeV}, v_{h}=v_{s}, \lambda_{s}=1 / 8$ and $\lambda_{h}=4 \lambda_{s}$; the intersection points of the curves correspond to the solution $\sin \chi=1 / 3$ for the mixing parameter.

The mixing is restricted by the positivity of the decay widths to the band

$$
\kappa_{2} \leq \sin ^{2} \chi \leq 1-\kappa_{1} .
$$

In Fig. 2 we show the numerical solutions to Eqs.(15) and (16). They return unique values of the mixing parameter $\sin ^{2} \chi$ and the partial widths $\Gamma_{1,2}^{\text {inv }}$ for decays of the two Higgs bosons $H_{1,2}$ to particles in the hidden sector. The parameters chosen in these figures are the same as for the Higgs masses in Fig. 2, supplemented for illustration by the identities $\Gamma_{1,2}^{\text {hid }} \equiv \Gamma_{1,2}^{S M}$.

Finalizing our argument, the set of phenomenological Higgs parameters is indeed sufficient for the reconstruction of the parameters in the Lagrangian:

$$
\left\{M_{1,2}, \sin \chi, \Gamma_{2}^{H H} ; M_{W}\right\} \quad \Longleftrightarrow \quad\left\{v_{s}, v_{h} ; \lambda_{s}, \lambda_{h} ; \eta_{\chi}\right\},
$$

where further details we give in Tab. I. The two values $\lambda_{s} v_{s}^{2}, \lambda_{h} v_{h}^{2}$ and $\eta_{\chi} v_{s} v_{h}$ [modulo the discrete sign ambiguity] can be derived from the measured Higgs boson masses $M_{1,2}$ and the mixing parameter $\tan ^{2} 2 \chi$. The vacuum expectation value $v_{s}$ is directly related to the $W$ boson mass $M_{W}=g v_{s} / 2$, while the only remaining free parameter $v_{h}$ is fixed by measuring the magnitude of the Higgs self-coupling $\Lambda_{211}$. A quantitative phenomenological example for reconstructing the theory this way we discuss in the following section.

\section{HIGGS PROFILING AT THE LHC}

The phenomenological analysis of the hidden-Higgs theory, after the discovery of the Higgs boson(s), splits into two parts. First, we determine the parameter regions where two, one or none of the Higgs particles can be detected. Subsequently, we study to which extent we can reconstruct the parameters of the standard and hidden Higgs sectors at the LHC.

The analyses will be exemplified by choosing the same value for $\Gamma^{\mathrm{SM}}$ as for the invisible width $\Gamma^{\text {hid }}$, which encodes the dynamics of the hidden sector. To asses the sensitivity of the LHC experiments at a typical SM Higgs discovery luminosity

\begin{tabular}{c|c|c}
\hline & parameters & measurements \\
\hline \multirow{2}{*}{ Higgs properties } & $M_{1,2}$ & resonance peaks \\
& $\sin ^{2} \chi, \Gamma_{1,2}^{\mathrm{inv}}$ & $\kappa_{1,2},\left\langle\sigma \mathrm{BR}^{\mathrm{inv}}\right\rangle$ \\
\hline \multirow{4}{*}{ Higgs potential } & $\lambda_{s} v_{s}^{2}, \lambda_{h} v_{h}^{2}, \eta_{\chi} v_{s} v_{h}$ & $M_{1,2}, \sin ^{2} \chi$ \\
& $v_{s}$ & $M_{W}$ \\
& $v_{h}$ & $\Gamma_{2}^{H H}$ \\
& $\Downarrow$ & \\
& $\lambda_{s}, \lambda_{h}, \eta_{\chi}$ & derived subsequently \\
\hline
\end{tabular}

TABLE I: Consecutive determination of the Higgs properties and parameters of the Higgs portal potential. 


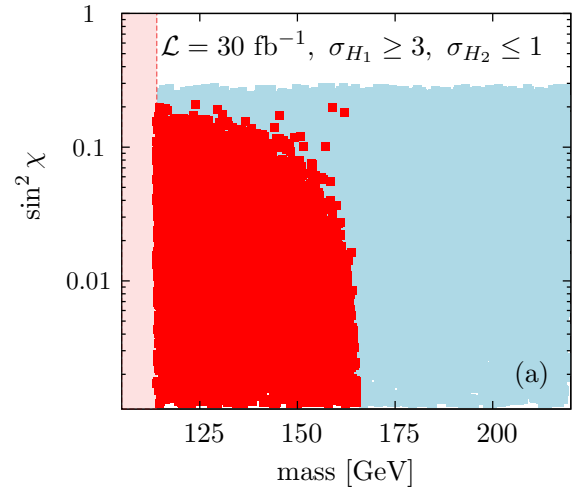

light SM-like Higgs $H_{1}$ excluded by EWP, unitarity excluded by LEP

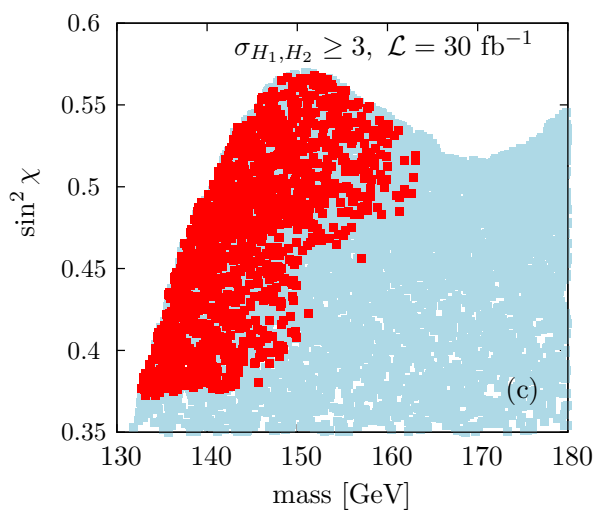

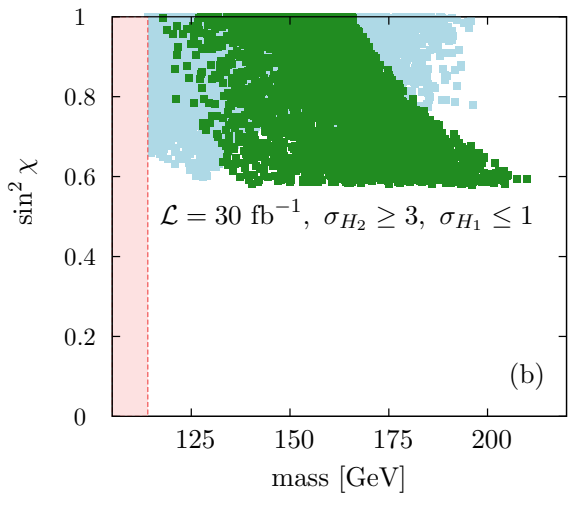

heavy dark Higgs $H_{2}$ excluded by EWP, unitarity excluded by LEP

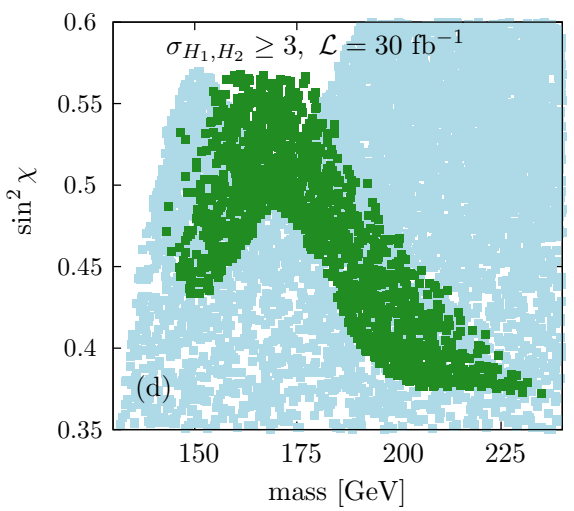

FIG. 3: Randomly generated Higgs portal models. The parameters ranges are $v_{h} \in(0 \mathrm{GeV}, 246 \mathrm{GeV}], v_{s}=246 \mathrm{GeV}, \lambda_{h} \in(0,4 \pi], \lambda_{s} \in$ $(0,4 \pi]$, and $\eta_{\chi} \in[-4 \pi, 4 \pi]$. The hidden Higgs decay width we identify with the SM decay width, i.e. $\Gamma^{\text {hid }} \equiv \Gamma^{S M}$. LEP constraints and bounds from $S, T, U$ [29] are included, likewise the unitarity constraint of Eq. (7). Panel (a) displays the sensitivity for $H_{1}$ only, panel (b) for $\mathrm{H}_{2}$ only, and panels $(c)$ and $(d)$ show where the LHC is sensitive to both $\mathrm{H}_{1}$ and $\mathrm{H}_{2}$ at the same time for $30 \mathrm{fb}^{-1}$. Cross sections and widths we compute using HIGLU [36] and HDECAY [37].

$\mathcal{L}=30 \mathrm{fb}^{-1}$ we randomly scan over the (hidden) Higgs potential of Eq. (1). Statistical significances we derive by rescaling the results of the experimental simulations in Ref. [25] for parameter choices consistent with the constraints from unitarity and electroweak precision data [29]. We choose $(S, T)=(0,0)$ for $m_{t}=170.9 \mathrm{GeV}$ [34] and $m_{H}^{\mathrm{SM}}=115 \mathrm{GeV}$. We have verified our implementation against Ref. [3].

First, we identify the part of parameter space where at least one Higgs boson can be discovered at the LHC in a visible channel. In terms of the masses $M_{1,2}$ and the mixing parameter $\sin ^{2} \chi$ we show this area in Fig. 3(a,b). The light SM-type Higgs boson is clearly visible for small $\sin ^{2} \chi$, the hidden-type Higgs boson for large $\sin ^{2} \chi$. In Fig. 3(c,d) the parameter space of $M_{1}, M_{2}, \sin ^{2} \chi$ is shown in which both Higgs bosons can be detected at the LHC. Combining them, we can distinguish four different areas for $\sin ^{2} \chi$ where either none or one or two Higgs bosons are accessible at the LHC for $\mathcal{L}=30 \mathrm{fb}^{-1}$ :

\begin{tabular}{|c|c|c|}
\hline & \# observable Higgs bosons & \\
\hline $\sin ^{2} \chi \lesssim 0.2$ & 1 & SM-type Higgs $H_{1} \quad\left(\sigma_{H_{1}} \geq 3, \sigma_{H_{2}} \leq 1\right)$ \\
\hline $0.3 \lesssim \sin ^{2} \chi \lesssim 0.4$ & 0 & neither SM-type nor hidden-type Higgs $H_{1}, H_{2} \quad\left(\sigma_{H_{1}, H_{2}}<3\right)$ \\
\hline $0.4 \lesssim \sin ^{2} \chi \lesssim 0.6$ & 2 & $\mathrm{SM}+$ hidden-type Higgs $H_{1}, H_{2} \quad\left(\sigma_{H_{1}, H_{2}} \geq 3\right)$ \\
\hline $\sin ^{2} \chi \gtrsim 0.6$ & 1 & hidden-type Higgs $H_{2} \quad\left(\sigma_{H_{1}} \leq 1, \sigma_{H_{2}} \geq 3\right)$ \\
\hline
\end{tabular}

When we steadily increase the hidden widths the fraction of parameter space in which none of the Higgs bosons can be detected increases. Thus, overwhelming decay modes into the hidden sector can screen Higgs bosons in a natural way [and non-observation of Higgs bosons, for limited luminosity, does not necessarily imply the falsification of the Higgs mechanism]. 
By measuring the production of the two Higgs bosons in the visible and invisible decay channels, we can determine the properties of the Higgs bosons, as demonstrated in the previous section: the masses $M_{1,2}$; the mixing parameter sin ${ }^{2} \chi$; and the invisible widths $\Gamma_{1,2}^{\text {inv }}$. Since invisible channels are involved, the full capacity of LHC with luminosity of $300 \mathrm{fb}^{-1} \mathrm{will}^{\mathrm{be}}$ needed to draw a finely-grained picture. The analysis would be redundant if the two Higgs bosons had been distinguishable in the invisible channel, which turns out to be a very difficult task.*

Fortunately, the parameters can also be determined if only the superposition of the two Higgs bosons is measured in the invisible channels. We define the weighted ratio of invisible over visible branching ratios by

$$
\left\langle\frac{\sigma \mathrm{BR}^{\mathrm{inv}}}{\sigma \mathrm{BR}^{\mathrm{vis}}}\right\rangle=\frac{\left\langle\sigma_{1} \mathrm{BR}_{1}^{\mathrm{inv}}+\sigma_{2} \mathrm{BR}_{2}^{\text {inv }}+\mathrm{Bkg}\right\rangle_{\text {inv }}}{\left\langle\sigma_{1} \mathrm{BR}_{1}^{\text {vis }}+\sigma_{2} \mathrm{BR}_{2}^{\text {vis }}+\mathrm{Bkg}\right\rangle_{\text {vis }}},
$$

where the subscripts 'inv' and 'vis' refer to different analysis strategies in measuring visible and invisible decays [25]. The production cross sections and branching ratios of the signal we can re-express in terms of the $\kappa_{1,2}$ parameters and the mixing angle:

$$
\begin{aligned}
\sigma_{j} \mathrm{BR}_{j}^{\mathrm{inv}} & =\sigma_{j}^{\mathrm{SM}}\left[\left\{\begin{array}{l}
\cos ^{2} \chi \\
\sin ^{2} \chi
\end{array}\right\}-\kappa_{j}\left(1+h_{j=2}^{\mathrm{inv}}\right)\right] \quad \text { for } j=1,2 \\
\sigma_{j} \mathrm{BR}_{j}^{\mathrm{vis}} & =\sigma_{j}^{\mathrm{SM}} \kappa_{j} .
\end{aligned}
$$

The $h$ term denotes generally small complements from $H_{2} \rightarrow H_{1} H_{1}$ cascade decays, which can be measured in the visible channels: $h_{2}^{\text {inv }}=-\kappa_{2}\left[\mathrm{BR}_{2}^{H H, \mathrm{vis}} / \mathrm{BR}_{2}^{\mathrm{vis}}\right]\left(1+\sin ^{2} \chi\right) / \cos ^{2} \chi$. Since the invisible and visible channels are treated incoherently, the measurement errors can be slightly reduced by restricting the analysis to the invisible decay modes $\left\langle\sigma \mathrm{BR}^{\text {inv }}\right\rangle$ instead of the ratio, $c f$. Fig. 4.

We adopt the experimental simulations of Ref. [25] for both Higgs bosons. Since the $\chi$ dependence of the cross section for invisible decays of the two-Higgs system is predicted to be

$$
\left\langle\sigma \mathrm{BR}^{\mathrm{inv}}\right\rangle \sim \text { const }-\left[\cos ^{2} \chi+\left\{\sigma_{2}^{\mathrm{SM}} / \sigma_{1}^{\mathrm{SM}}\right\} \sin ^{2} \chi\right]
$$

we lose all sensitivity if the two Higgs masses are too close and the ratio of the two SM cross sections is near unity. However, for increasing $\mathrm{H}_{2}$ masses, the $\mathrm{H}_{2}$ contribution steadily drops with the production cross section. This leads to the asymptotic behavior $\langle\sigma \mathrm{BR}$ inv $\rangle \sim$ const $-\cos ^{2} \chi$ generated by $H_{1}$ alone; potential errors induced by the extrapolation in the $H_{2}$ mass are suppressed as a result.

The cross section $\left\langle\sigma \mathrm{BR}^{\text {inv }}\right\rangle$ for invisible decays we illustrate in Fig. 4 for three reference points in which invisible and visible decays of the two Higgs bosons are nearly balanced. The reference points are characterized by the $H_{1}, H_{2}$ masses and the true mixing angle:

$$
\begin{array}{ll}
\text { ref. point \#1 } & \left\{M_{1}, M_{2} ; \sin ^{2} \chi\right\}=\{140,160 ; 0.456\}, \\
\text { ref. point \#2 } & \left\{M_{1}, M_{2} ; \sin ^{2} \chi\right\}=\{115,300 ; 0.25\}, \\
\text { ref. point \#3 } & \left\{M_{1}, M_{2} ; \sin ^{2} \chi\right\}=\{115,400 ; 0.25\},
\end{array}
$$

with masses in units of GeV. For these parameter points the discovery of the Higgs bosons is steered by the visible decay channels $H_{1} \rightarrow b \bar{b}, \gamma \gamma\left(M_{1}=115 \mathrm{GeV}\right)$ and $H_{i} \rightarrow 4 \ell\left(M_{1}=140 \mathrm{GeV}, M_{2}=300,400 \mathrm{GeV}\right)$. Ref. point \#3 falls mildly outside the 95\% confidence level $S, T$ contour: $S=0.018, T=-0.031<T_{95 \%}=-0.028$. It exemplifies the asymptotic behavior of a large hierarchy between the two production rates $\sigma_{2}^{\mathrm{SM}} / \sigma_{1}^{\mathrm{SM}} \sim 3 \mathrm{fb} / 44 \mathrm{fb}$. The production rates include gluon fusion [35] as cast in HIGLU [36] and electroweak vector boson fusion VBFNLO [22]; branching ratios for decay channels we adopt from HDECAY [37]. Background estimates we incorporate by adjusting the simulations described in Ref. [25]. From the three measurement shown, the value of $\sin ^{2} \chi$ can be determined uniquely. Given this mixing parameter, we can finally calculate the partial widths for invisible Higgs decays into the hidden sector, as shown in Eqs. (15) and (16). The result, including only statistical effects on the extraction of $\sin ^{2} \chi$ we show in Tab. II.

We see that depending on the specific realization of the Higgs portal we can reconstruct some, if not all, elements of the underlying theory, i.e. the vacuum expectation values and quartic couplings in the standard and hidden sectors, as well as the

\footnotetext{
${ }^{*}$ Varying the LHC energy from 7 to $14 \mathrm{TeV}$, the $H_{1}$ and $H_{2}$ components may in principle be disentangled. Missing mass techniques in $e^{+} e^{-}$collisions, $c f$.
} Ref. [38], allow for a separation of the two Higgs bosons also in invisible decays. 

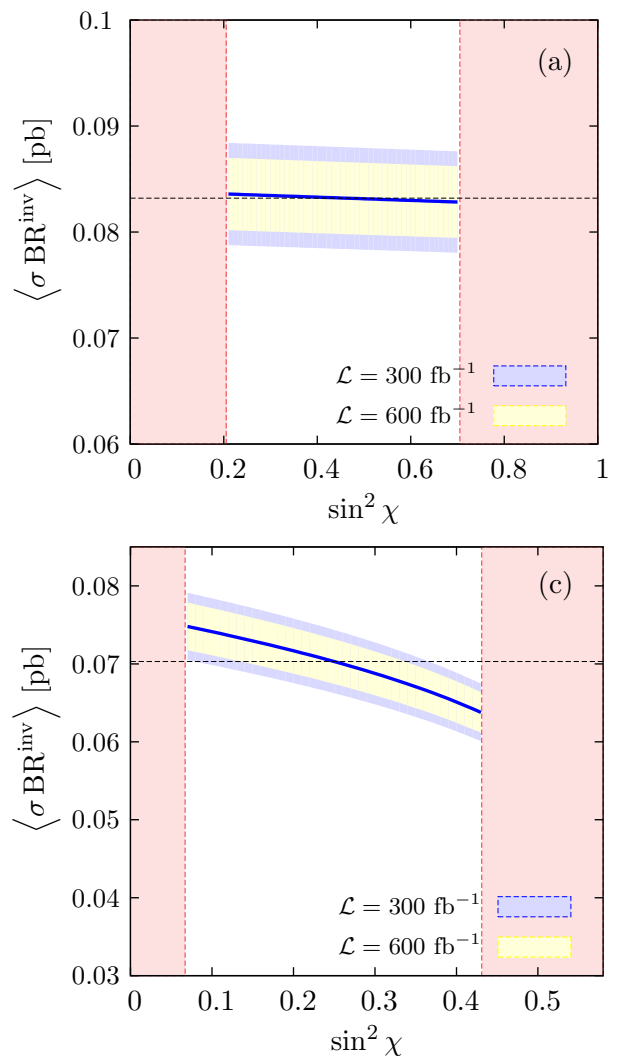

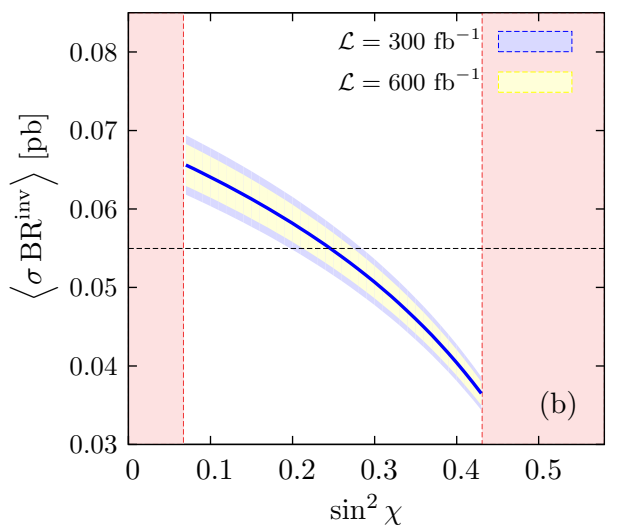

FIG. 4: (a) $\left\langle\sigma B R^{i n v}\right\rangle$ corresponding to reference point \#1. (cf. Tabs. II and III). Both Higgs bosons exhibit significances of $3 \sigma$ or more in the visible sector for $\mathcal{L}=$ $30 \mathrm{fb}^{-1}$ and will be well-established with $\mathcal{L}=300,600 \mathrm{fb}^{-1}$. The dotted line gives the true Monte Carlo value. (b) and (c) Extracting the mixing parameters in the reference points \#2 and \#3, respectively.

coupling of the Higgs portal. If the invisible decays of the heavier state $H_{2}$ can be decoupled from $\left\langle\sigma \mathrm{BR}^{\text {inv }}\right\rangle$ by suppressing the production cross section, Eq. (20), the value of $\sin ^{2} \chi$ can eventually be inferred from the invisible Higgs decays. The measurement of the self-coupling $\Lambda_{211}$ provides additional information, which further constrains the allowed parameters of the hidden sector. After $\lambda_{h} v_{h}^{2}$ is determined by measuring the Higgs masses and the mixing parameter, the coupling $\lambda_{h}$ and the vacuum expectation value $v_{h}$ can be disentangled in cascade decays.

Quantitatively, the determination of the mixing angle and the separation of the two invisibly decaying Higgs states can vary significantly. For the reference point \#1 the two Higgs masses are close to each other, so the SM production cross sections are almost the same and the sum of both invisible production and decay rates is nearly independent of the mixing parameter. This is reflected in the almost horizontal curves in Fig. 4(a). In addition, the measurement of the self-coupling is very difficult

\begin{tabular}{l|c|c|c}
\hline & reference point \#1 & reference point \#2 & reference point \#3 \\
\hline $\sin ^{2} \chi$ & $0.46 \pm 0.25$ & $0.24 \pm 0.04(0.25 \pm 0.03)$ & $0.22 \pm 0.15(0.24 \pm 0.10)$ \\
$\Gamma_{1}^{\text {inv }} / \Gamma_{1}^{S M}$ & $0.46 \pm 0.67$ & $0.25 \pm 0.07(0.25 \pm 0.05)$ & $0.29 \pm 0.26(0.27 \pm 0.17)$ \\
$\Gamma_{2}^{\text {inv }} / \Gamma_{2}^{S M}$ & $0.54 \pm 0.83$ & $2.86 \pm 0.74(2.89 \pm 0.54)$ & $0.01 \pm 0.29(0.02 \pm 0.23)$ \\
\hline
\end{tabular}

TABLE II: Parameters extracted for the three reference points defined in Eq. (21); the luminosity is set to $\mathcal{L}=300 \mathrm{fb}^{-1}\left(600 \mathrm{fb} b^{-1}\right)$. For reference point \#1 increased statistics does not amount to smaller errors since the two states $H_{1}, H_{2}$ are too close in mass.

\begin{tabular}{l|cc|ccc|ccc}
\hline & \multicolumn{2}{|c|}{ reference point \#1 } & \multicolumn{3}{|c|}{ reference point \#2 } & \multicolumn{3}{|c}{ reference point \#3 } \\
\hline$v_{s}[\mathrm{GeV}]$ & \multicolumn{2}{|c|}{246.22} & \multicolumn{3}{c|}{246.22} & \multicolumn{3}{c}{246.22} \\
$\lambda_{s}$ & $0.18 \pm 0.01$ & {$[0.19]$} & $0.58 \pm 0.03$ & $(0.58 \pm 0.02)$ & {$[0.58]$} & $1.04 \pm 0.18$ & $(1.03 \pm 0.12)$ & {$[1.03]$} \\
$v_{h}[\mathrm{GeV}]$ & $85.72 \pm 32.88$ & {$[85.75]$} & $36.19 \pm 5.06$ & $(36.42 \pm 3.63)$ & {$[36.42]$} & $55.03 \pm 27.35$ & $(58.11 \pm 18.94)$ & {$[60.28]$} \\
$\lambda_{h}$ & $1.53 \pm 0.10$ & {$[1.52]$} & $12.21 \pm 1.25$ & $(12.12 \pm 0.89)$ & {$[12.11]$} & $7.61 \pm 3.51$ & $(7.19 \pm 2.23)$ & {$[6.97]$} \\
$\left|\eta_{\chi}\right|$ & $0.13 \pm 0.40$ & {$[0.13]$} & $3.67 \pm 0.53$ & $(3.66 \pm 0.38)$ & {$[3.61]$} & $4.52 \pm 2.23$ & $(4.38 \pm 1.50)$ & {$[4.40]$} \\
\hline
\end{tabular}

TABLE III: Extracted Lagrangian parameters of the standard sector, the hidden sector, and their coupling for $\mathcal{L}=300 \mathrm{fb}^{-1}\left(600 \mathrm{fb}^{-1}\right)$. Not included are uncertainties that arise from measurements of the masses $W, H_{1}$, and $H_{2}$ and the coupling $\Lambda_{211}$. The values in squared brackets are the true input. 
as the decay of the light $H_{2}$ to two on-shell $H_{1}$ particles is kinematically forbidden. On the other hand, for sufficiently large $H_{1}, H_{2}$ mass splittings and away from maximal mixing $\sin ^{2} \chi \approx \cos ^{2} \chi \approx 0.5$, as realized in the reference points \#2 and \#3, these shortcomings are largely removed ${ }^{\dagger}$ : since the SM cross section $\sigma_{2}^{\mathrm{SM}}$ is non-dominant, the cross section for invisible Higgs decays does depend on the mixing parameter. As a result, we can determine $\sin ^{2} \chi$, as shown in Fig. 4(b,c). In addition, on-shell cascade decays $H_{2} \rightarrow H_{1} H_{1}$ open and can be exploited to determine $\Lambda_{211}$. In such cases, Tab. III shows that the Higgs potential parameters of the Higgs portal can be determined in toto: The extraction of the parameters improves upon increased statistics.

\section{SUMMARY}

A two-state Higgs portal has exciting experimental implications for the LHC. Depending on the parameters, three scenarios in which no or one or two Higgs bosons are observed could be realized.

- Even though two Higgs bosons are incorporated in the system, for medium size mixing none would be observed. This leads to the startling conclusion that non-observation of Higgs bosons, for luminosities typical for the discovery of the standard Higgs boson, does not imply that the Higgs mechanism is falsified.

- If one Higgs boson is observed experimentally, the couplings must be investigated to conclude that the standard mechanism is realized. Mixing of the states in the Higgs portal predicts the uniform reduction of the couplings to the particles in the Standard Model. Enhanced by decays into the hidden sector, the decay properties and the production rates are affected, resulting in the universal reduction of the twin-width ratios of partial widths over the total width which can be observed directly at LHC.

- Finally, if two Higgs bosons are detected, subsequently to the profile of the particles, masses and mixing, the microscopic elements of the Higgs portal can in principle be reconstructed. This is a difficult experimental task since control over invisible channels and two-Higgs final states is necessary for drawing a fine-grained picture. Nevertheless, in this situation we can determine the vacuum expectation values of the two Higgs fields, the two quartic couplings of the fields in the standard and the hidden sector as well as the quartic coupling of the two Higgs fields across the portal.

Thus, the Higgs portal including a two-state system offers on one the hand a simple but interesting extension of the SM Higgs mechanism; on the other hand, it leads to an exciting lookout to a novel hidden sector in nature, which can phenomenologically be accessed at the LHC.

\section{Acknowledgments}

PMZ thanks the Institut für Theoretische Teilchenphysik und Kosmologie for the warm hospitality extended to him at RWTH Aachen University. DZ und PMZ are grateful to the Institut für Theoretische Physik of Heidelberg University for the hospitality during several visits. Part of the work by DZ is supported by the GDR Terascale of the CNRS.

[1] D. E. Morrissey, T. Plehn and T. M. P. Tait, arXiv:0912.3259 [hep-ph]; J. D. Wells, arXiv:0803.1243 [hep-ph].

[2] R. Schabinger and J. D. Wells, Phys. Rev. D 72 (2005) 093007.

[3] M. Bowen, Y. Cui and J. D. Wells, JHEP 0703 (2007) 036.

[4] R. Foot, H. Lew and R. R. Volkas, Phys. Lett. B 272 (1991) 67; R. Foot, H. Lew and R. R. Volkas, Mod. Phys. Lett. A 7 (1992) 2567.

[5] Z. Chacko, H. S. Goh and R. Harnik, Phys. Rev. Lett. 96 (2006) 231802.

[6] R. Barbieri, T. Gregoire and L. J. Hall, arXiv:hep-ph/0509242.

[7] B. Patt and F. Wilczek, arXiv:hep-ph/0605188.

[8] M. J. Strassler and K. M. Zurek, Phys. Lett. B 651, 374 (2007); M. J. Strassler and K. M. Zurek, Phys. Lett. B 661, 263 (2008).

[9] V. Barger, P. Langacker, M. McCaskey, M. J. Ramsey-Musolf and G. Shaughnessy, Phys. Rev. D 77, 035005 (2008).

\footnotetext{
${ }^{\dagger}$ For the light Higgs mass chosen in this study, we assume the cascade $H_{2} \rightarrow H_{1} H_{1}$ to be reconstructed from the $H_{1} \rightarrow \gamma \gamma$, which, however, has a smaller branching ratio than the competing channel $H_{1} \rightarrow b \bar{b}$ but similar significance [25]. The subjet analysis as considered in Ref. [39] could, eventually, allow the reconstruction $H_{2} \rightarrow H_{1} H_{1} \rightarrow b \bar{b} b \bar{b}, 4 g, \tau \bar{\tau} \tau \bar{\tau}$. It should be noted that a large fraction of $H_{2}$ Higgs bosons decay to $H_{1} H_{1}$ pairs, facilitating the measurement of the $H_{2} H_{1} H_{1}$ coupling compared with the measurement of the Higgs trilinear coupling in the Standard Model.
} 
[10] O. Lebedev and H. M. Lee, arXiv:1105.2284 [hep-ph].

[11] W. F. Chang, J. N. Ng and J. M. S. Wu, Phys. Rev. D 74 (2006) 095005 [Erratum-ibid. D 79 (2009) 039902]; W. F. Chang, J. N. Ng and J. M. S. Wu, Phys. Rev. D 75 (2007) 115016

[12] S. Kanemura, S. Matsumoto, T. Nabeshima and N. Okada, Phys. Rev. D 82 (2010) 055026.

[13] S. Bock, R. Lafaye, T. Plehn, M. Rauch, D. Zerwas and P. M. Zerwas, Phys. Lett. B 694 (2010) 44.

[14] T. Binoth and J. J. van der Bij, Z. Phys. C 75, 17 (1997).

[15] M. Dührssen, S. Heinemeyer, H. Logan, D. Rainwater, G. Weiglein and D. Zeppenfeld, Phys. Rev. D 70, 113009 (2004).

[16] R. Lafaye, T. Plehn, M. Rauch, D. Zerwas and M. Dührssen, JHEP 0908, 009 (2009).

[17] A. Dedes, T. Figy, S. Hoche, F. Krauss and T. E. J. Underwood, JHEP 0811 (2008) 036.

[18] A. Djouadi, Phys. Rept. 457, 1 (2008); Phys. Rept. 459, 1 (2008).

[19] M. Spira, Fortsch. Phys. 46, 203 (1998); V. Buescher and K. Jakobs, Int. J. Mod. Phys. A 20, 2523 (2005); D. Rainwater, [arXiv:hepph/0702124]; M. Gomez-Bock, M. Mondragon, M. Mühlleitner, M. Spira and P. M. Zerwas, [arXiv:0712.2419 [hep-ph]]; J. D. Wells, [arXiv:0909.4541 [hep-ph]]; T. Plehn, [arXiv:0910.4182 [hep-ph]].

[20] A. Djouadi, W. Kilian, M. Mühlleitner and P. M. Zerwas, Eur. Phys. J. C 10 (1999) 27; C. Castanier, P. Gay, P. Lutz and J. Orloff, arXiv:hep-ex/0101028; U. Baur, T. Plehn, D. L. Rainwater, Phys. Rev. Lett. 89 (2002) 151801, Phys. Rev. D68 (2003) 033001, and Phys. Rev. D69 (2004) 053004.

[21] D. L. Rainwater, D. Zeppenfeld and K. Hagiwara, Phys. Rev. D 59 (1998) 014037; T. Plehn, D. L. Rainwater and D. Zeppenfeld, Phys. Rev. D 61 (2000) 093005; N. Kauer, T. Plehn, D. L. Rainwater and D. Zeppenfeld, Phys. Lett. B 503 (2001) 113.

[22] K. Arnold et al., Comput. Phys. Commun. 180 (2009) 1661.

[23] T. Plehn, G. P. Salam, M. Spannowsky, Phys. Rev. Lett. 104 (2010) 111801.

[24] O. J. P. Eboli and D. Zeppenfeld, Phys. Lett. B 495 (2000) 147.

[25] G. Aad et al. [The ATLAS Collaboration], arXiv:0901.0512 [hep-ex]; A. De Roeck et al., Eur. Phys. J. C 66 (2010) 525.

[26] J. Bagger et al., Phys. Rev. D 52 (1995) 3878; C. Englert, B. Jager, M. Worek and D. Zeppenfeld, Phys. Rev. D 80 (2009) 035027.

[27] R. Barate et al. [LEP Working Group for Higgs boson searches and ALEPH, DELPHI, L3 and OPAL Collabs], Phys. Lett. B 565 (2003) 61.

[28] M. E. Peskin and T. Takeuchi, Phys. Rev. Lett. 65 (1990) 964.

[29] J. Alcaraz et al. [ALEPH Collaboration and DELPHI Collaboration and L3 Collaboration and ], arXiv:hep-ex/0612034, [see also http: // lepewwg.web.cern.ch/LEPEWWG/plots/summer2006/]; J. Erler and P. Langacker in K. Nakamura et al. [Particle Data Group], J. Phys. G 37 (2010) 075021.

[30] M. E. Peskin and J. D. Wells, Phys. Rev. D 64 (2001) 093003.

[31] M. Ahlers, J. Jaeckel, J. Redondo, A. Ringwald, Phys. Rev. D78 (2008) 075005. [arXiv:0807.4143 [hep-ph]].

[32] B. Holdom, Phys. Lett. B 166 (1986) 196; K. L. McDonald, D. E. Morrissey, JHEP 1102 (2011) 087.

[33] G. F. Giudice, C. Grojean, A. Pomarol and R. Rattazzi, JHEP 0706 (2007) 045; J. R. Espinosa, C. Grojean and M. Mühlleitner, JHEP 1005 (2010) 065; R. Grober and M. Mühlleitner, arXiv:1012.1562 [hep-ph].

[34] CDF and D0 Collaboration, arXiv:hep-ex/0703034.

[35] M. Spira, A. Djouadi, D. Graudenz and P. M. Zerwas, Nucl. Phys. B 453 (1995) 17.

[36] M. Spira, Nucl. Instrum. Meth. A 389, 357 (1997).

[37] A. Djouadi, J. Kalinowski and M. Spira, Comput. Phys. Commun. 108 (1998) 56.

[38] W. Kilian and P. M. Zerwas, ILC: Physics scenarios, Proceedings, LC Workshop, Snowmass 2005, arXiv:hep-ph/0601217.

[39] J. M. Butterworth, A. R. Davison, M. Rubin and G. P. Salam, Phys. Rev. Lett. 100 (2008) 242001; A. Falkowski, D. Krohn, L. T. Wang, J. Shelton and A. Thalapillil, arXiv:1006.1650 [hep-ph]; C. R. Chen, M. M. Nojiri and W. Sreethawong, JHEP 1011 (2010) 012; A. Martin and T. S. Roy, arXiv:1103.3504 [hep-ph]; C. Englert, T. S. Roy and M. Spannowsky, arXiv:1106.4545 [hep-ph]. 
